\title{
How did COVID-19 impact on dental antibiotic prescribing across England?
}

\author{
Sagar Shah, ${ }^{1}$ Valerie Wordley² and Wendy Thompson*3
}

\section{Key points}

Restricted access to dentistry due to COVID-19 has resulted in increased dental antibiotic prescribing, which peaked in June 2020
The impact of COVID-19 has affected the rate of dental antibiotic prescribing differently across the regions of England.
Dentistry has an essential role to play in tackling antibiotic resistance by optimising antibiotic use. Introducing e-prescribing will be an important step to enable monitoring of dental antibiotic prescribing.

\begin{abstract}
Introduction Antibiotic resistance is a global problem driven by unnecessary antibiotic use. Between 25 March-8 June 2020, COVID-19 restrictions severely reduced access to dentistry in England. Dental practices were instructed to manage patients remotely with advice, analgesics and antibiotics, where appropriate.
\end{abstract}

Aim To describe the impact of the policy to restrict dental access on antibiotic prescribing.

Methods NHS Business Services Authority 2018-2020 data for England were analysed to describe national and regional trends in dental antibiotic use.

Results Antibiotic prescribing in April to July 2020 was 25\% higher than April to July 2019, with a peak in June 2020. Some regions experienced greater increases and for longer periods than others. The increase was highest in London $(60 \%)$ and lowest in the South West (10\%). East of England had the highest rate of dental antibiotic prescriptions per 1,000 of the population every month over the study period (April to July 2020).

Conclusion Restricted access to dental care due to COVID-19 resulted in greatly increased dental antibiotic prescribing, against an otherwise downward trend. As dental care adapts to the COVID-19 era, it is important to ensure access for all to high-quality urgent dental care. Understanding the reasons for variation will help to optimise the use of antibiotics in the future.

\section{Introduction}

Antibiotic resistance is a major public health burden driven by the use of antibiotics. ${ }^{1}$ Severely restricted access to dental care during the early stages of the COVID-19 pandemic led to a significant increase in antibiotic prescribing by dentists. ${ }^{2}$ On 25 March 2020, dental practices in England were instructed to suspend all routine, non-urgent dental care and all emergency dental care was provided through a network of local National Health

${ }^{1}$ Clinical Fellow, NHS Business Services Authority, $1 \mathrm{St}$ Anne's Road, Eastbourne, East Sussex, BN21 3UN, UK

${ }^{2} \mathrm{NHS}$ England, London, SE1 6LH, UK; ${ }^{3} \mathrm{NIHR}$ Clinical Lecture in Primary Dental Care, Division of Dentistry, University of Manchester, Couplands 3, Oxford Road, Manchester, M13

9PL, UK.

${ }^{\star}$ Correspondence to: Wendy Thompson

Email address: wendy.thompson15@nhs.net

Refereed Paper.

Accepted 14 October 2020

https://doi.org/10.1038/s41415-020-2336-6
Service (NHS) urgent dental care systems. ${ }^{3}$ Dental practices were restricted to providing remote management of patients by telephone and were advised to provide advice, analgesics and antibiotics (the AAA approach), where appropriate. ${ }^{3}$ On 8 June, practices were permitted to commence reopening for face-toface care while ensuring adherence to COVIDrelated safety measures. ${ }^{4}$ This resumption of services occurred over a period of weeks and concerns have been raised about the backlog of routine dental care which accrued during the period of restricted access. ${ }^{5}$ A report by the UK Parliament's House of Commons Health and Social Care Committee about the impact of the COVID-19 pandemic on NHS services states: 'patients have been remotely prescribed with antibiotics for their dental problems but have returned with pain or further swelling as the cause of their dental problem has not been properly addressed...this is contributing to an "overhang of oral healthcare".
This confirms the adage 'antibiotics do not cure toothache.' Yet, before COVID-19, high rates of dental antibiotic overprescribing were identified, with $80 \%$ unnecessary use (not in accordance with guidance) reported in both the $\mathrm{UK}^{6}$ and US. ${ }^{7}$ Dental guidelines for the treatment of dental infections are generally based on the principle of draining infections and removing the cause, which may necessitate a dental extraction or extirpation. ${ }^{8,9}$ Antibiotics are usually reserved for the treatment of severe dental infections (such as extraoral facial swelling) with systemic complications (such as pyrexia). ${ }^{10}$ In the absence of infection, antibiotics are inappropriate, yet studies and audits consistently show that dentists prescribe them for conditions such as irreversible pulpitis. ${ }^{11,12,13,14}$ A variety of approaches to optimise dental antibiotic prescribing across England have been developed and, in 2016, they were brigaded into a dedicated, national, online dental antimicrobial stewardship toolkit. ${ }^{15}$ 
A downward trend in dental antibiotic use since 2011 has been reported in England. ${ }^{16}$

With restricted access to face-to-face dental services during the COVID-19 pandemic and the AAA approach advocated for remote management of patients, the aim of this study was to describe the impact of the policy to restrict access to dental services in England during the COVID-19 pandemic on NHS dental antibiotic prescribing.

\section{Method}

The objectives of this study were twofold: to describe trends in overall dental antibiotic use across England between January 2018 and July 2020; and to compare trends in the rate of use between the seven NHS England regions, from when restrictions were first placed on face-toface dental care to the most up-to-date data available (April 2020 to July 2020).

\section{Datasets}

The number of antibiotic items (BNF section 5.1 - 'antibacterial drugs') dispensed by community pharmacists in England relating to NHS dental prescription (FP10D) forms from January 2018 to July 2020 were provided by the NHS Business Services Authority (NHSBSA). ${ }^{17,18}$ The datasets exclude prescriptions dispensed from prisons, hospitals and private prescriptions. The data are publicly available from the NHS under the UK Freedom of Information Act 2000. The NHSBSA datasets are under Crown copyright and their use is licensed under the terms of the 'Open government licence for public sector information'. ${ }^{19}$

The mid-year population estimates were obtained from the UK Office for National Statistics. ${ }^{20}$ As population estimates for 2020 had not been published at the time of the analysis, population figures for 2019 were used for calculating the rates of dental antibiotic prescribing for 2020 .

As the data were obtained from national data resources and completely anonymised, ethical approval was not required.

\section{Outcomes reported}

Three outcomes are reported: 1) the total number of antibiotic prescriptions dispensed by community pharmacists to NHS dental patients in England each month between January 2018 and July 2020;2) the national rate of prescribing per 1,000 of the population; and 3 ) the monthly rate of prescribing per 1,000

Fig. 1 Number of antibiotic items dispensed by community pharmacists in England to NHS dental patients between January 2018 and July 2020. Data source: NHSBSA ${ }^{17}$

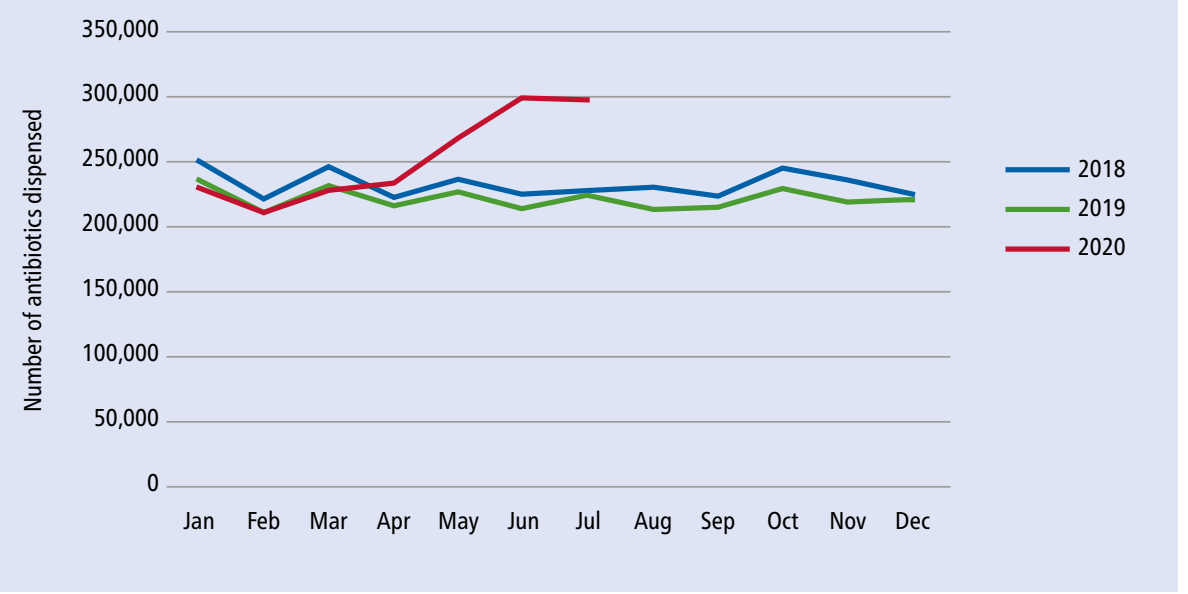

Table 1 Number and rate of antibiotic items dispensed by pharmacists to NHS dental patients in England during 2018 and 2019

\begin{tabular}{l|l|l|l}
\hline Statistic & $\mathbf{2 0 1 8}$ & $\mathbf{2 0 1 9}$ & $\mathbf{2 0 2 0}$ (January to July) \\
\hline $\begin{array}{l}\text { Total number of antibiotics dispensed to NHS } \\
\text { dental patients }\end{array}$ & $2,779,750$ & $2,651,449$ & $1,762,368$ \\
\hline Population of England & $55,977,000$ & $56,286,961$ & $56,286,961^{*}$ \\
\hline $\begin{array}{l}\text { Rate of dental antibiotics per 1,000 of the } \\
\text { population (annual) }\end{array}$ & 49.7 & 47.1 & N/A** \\
\hline $\begin{array}{l}\text { Rate of dental antibiotics per 1,000 of the } \\
\text { population (monthly) }\end{array}$ & 4.1 & 3.9 & 4.5 \\
$\begin{array}{l}\text { Key: } \\
*=2019 \text { figure employed as the mid-year population estimate for 2020 is not yet available } \\
* *=\text { only part of a year so no annual rate calculated }\end{array}$
\end{tabular}

of the population for each of England's seven regions (East of England; London; Midlands; North East and Yorkshire; North West; South East; and South West).

\section{Results}

Total number of dental prescriptions dispensed

A downward trend in the number of antibiotic prescriptions dispensed to NHS dental patients was seen through the study period until March 2020 (see Figure 1). After dental practices were restricted to providing only remote management of patients with AAA in late March, a sharp upward trend occurred during the months of April to June 2020. After dental practices were able to reopen in June 2020, the number of antibiotic dispensing to NHS dental patients plateaued in July 2020. The total number of antibiotic items dispensed for the period of April to July was $25 \%$ higher in 2020 compared to 2019 $(1,095,486$ vs 878,993$)$.

\section{National prescribing rate 2018-2020}

The average monthly rate of NHS dental antibiotic prescriptions was 4.1 items dispensed per 1,000 of the population in 2018 (see Table 1). It reduced to 3.9 in 2019 and then increased to 4.5 for the period of January to July 2020 .

\section{National and regional prescribing rates since COVID-19 restrictions}

At the beginning of the period of COVID-19 restrictions (April 2020), the monthly rate of dental antibiotic prescriptions was 4.1 per 1,000 of the population across England, ranging from 3.4 prescriptions in London to 5.0 in the East of England region. At the end of the study period (July 2020), the monthly rate was 5.3 per 1,000 of the population across England, ranging from 4.3 prescriptions in the South West region to 5.8 in the East of England. The rate peaked at 5.31 in June (28\% higher than in April) before it reduced very slightly in July.

As shown in Figure 2, all areas saw an increase in May and June 2020. Two regions (London and South East) continued to rise in 
Fig. 2 Rate of antibiotic items dispensed per 1,000 of the population to NHS dental patients by community pharmacists across England's seven regions between April and July 2020. Data source: NHSBSA and UK Office for National Statistics ${ }^{18,20}$

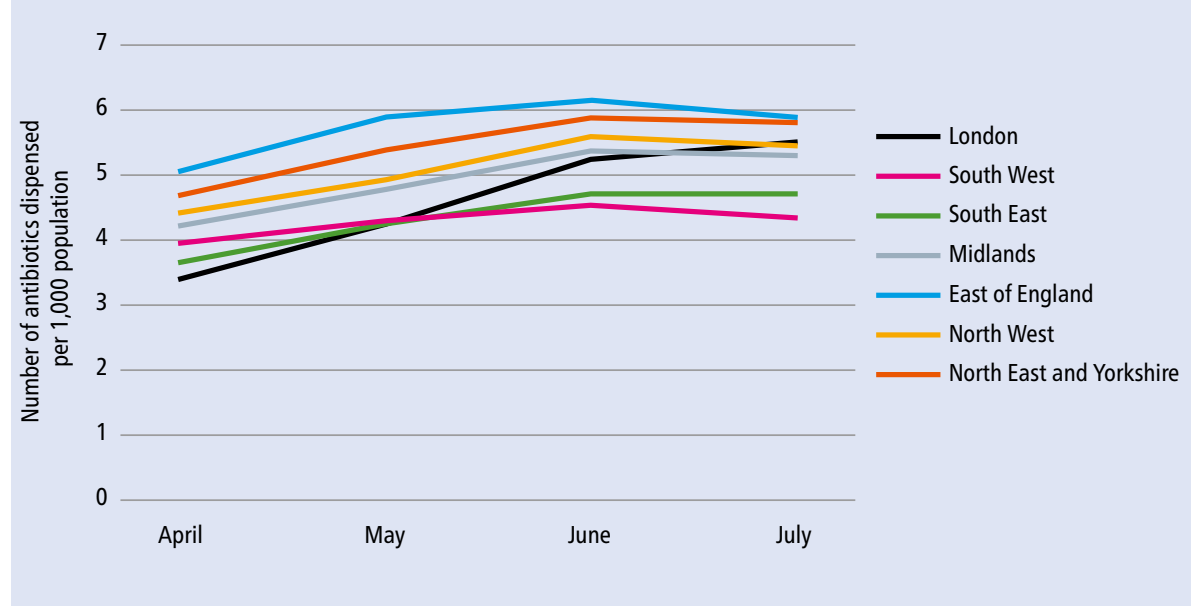

July, albeit the rate of increase slowed. Overall, the highest rate of antibiotic dispensing to NHS dental patients occurred in East of England during June (6.1 antibiotic prescriptions per 1,000 of the population). The lowest rate was in London during April (3.4 antibiotics per 1,000 population). London also saw the highest increase, with just over $60 \%$ more antibiotics in July than April 2020. In July, the lowest rate was in the South West (4.3 per 1,000 population) which also saw the smallest increase between April and July (less than 10\%).

\section{Discussion}

Restricted access to dentistry due to COVID19 has resulted in increased dental antibiotic prescribing across England. The impact has affected antibiotic prescribing rates differently between the regions, with the peak rate higher and taking longer to occur in some regions. By July, the overall rate of increase nationally had plateaued and some of the regions had already returned to levels of antibiotic use below that in April. Dentistry has an essential role to play in tackling antibiotic resistance, including by reducing unnecessary antibiotic prescribing.

Unnecessary dental antibiotic prescribing is a complex behaviour which is influenced by a plethora of clinical and non-clinical factors which affect dentists and patients. ${ }^{21,22}$ Early in the COVID-19 pandemic, a selection of potential factors driving the increase in dental antibiotic use were identified in a letter to the British Dental Journal editor-in-chief. ${ }^{2}$ Access and other systems/process issues were hypothesised as key, noting in particular that some urgent dental centres had been requiring patients to have tried antibiotics before accepting referral for face-to-face care. Further research to interpret the variation found between the regions will follow.

Monitoring of dental antibiotic prescribing is an essential element of evaluating efforts to optimise dental antibiotic prescribing. No data of sufficient quality for this purpose is currently routinely collected in England at the level of individual dental practices, clinicians or patients. The introduction of e-prescribing for dentistry, as advocated by the National Institute for Health and Care Excellence in its 2016 Antimicrobial stewardship: quality standard, ${ }^{23}$ would facilitate this and should be an essential component of antibiotic stewardship approaches in the future.

A comparison of COVID-19's impact on dental antibiotic prescribing in other countries is not yet possible. It is anticipated, however, that countries where guidelines indicate prophylactic use of antibiotics by dentists to protect people who may be at risk of distant site infections (such as infective endocarditis) ${ }^{24,25}$ may see a net reduction in antibiotic prescribing related to a reduction in the provision of routine dental care. In England, levels of prophylactic dental antibiotic use are known to be extremely low, ${ }^{26}$ as guidelines do not recommend the routine use of prophylactic antibiotics. ${ }^{27}$ For this reason, England may see a larger increase in overall dental antibiotic prescribing than other countries due to minimal offset from reductions in prophylactic use from reduced routine dental care provision.

Even before COVID-19, there was evidence of significant overprescribing of dental antibiotics internationally. ${ }^{7,28,29,30}$ Approaches to address unnecessary and inappropriate antibiotic use are urgently required in dental practices. The FDI World Dental Federation white paper, published in November 2020, advocates three roles for dental teams in tackling antibiotic resistance: 1) raising awareness about antibiotic resistance; 2) preventing dental infections; and 3) antibiotic stewardship to optimise dental antibiotic prescribing in accordance with guidelines. National dental associations are encouraged to commit to supporting international efforts to tackle antibiotic resistance and to advocate for dentistry within national action plans. The British Dental Association already campaigns for improved provision of urgent dental care. ${ }^{31}$ The House of Commons Health and Social Care Committee now also clearly recognises that dental procedures, not antibiotic prescriptions, are required to address the cause of dental pain and swelling. ${ }^{5}$

\section{Limitations}

Aggregation and anonymisation of the datasets provided by NHSBSA means that it was not possible to determine the clinical indications/ appropriateness of the prescriptions, nor demographics of the patients who were receiving the antibiotics. Data of sufficient quality for analysis or monitoring of dental antibiotic prescribing at the level of individual dental practices, clinicians or patients are not routinely collected in England at this time.

During the period of increased dental restrictions owing to COVID-19 (25 March-8 June 2020), all patients in England requiring urgent dental care were treated within the NHS. As practices reopened, more and more dentistry would have been provided by private providers; as data for private prescriptions are not routinely collected in England, the antibiotic figures for June and July may be under-reported compared to the figures for April and May.

On 1 April 2020, NHS England reorganised its regions. As a result, it is not possible to directly compare regional rates of dental antibiotic prescribing before and after COVID19 restrictions.

\section{Conclusions}

Restricted access to dental care has resulted in increased use of antibiotics. The impact affected some regions more and for longer than others. Access to dental services is an important non-clinical factor which drives 
unnecessary dental antibiotic prescribing. Dentistry has an essential role to play in global efforts to tackle antibiotic resistance. In the words of the House of Commons Health and Social Care Committee, when people present with pain or swelling, dentists must 'properly address the cause of the problem.' As dental care provision adapts to the COVID-19 era, it is important to ensure access for all to highquality urgent dental care and to understand the reasons for variation in order to optimise the use of antibiotics in the future.

\section{Acknowledgements}

No funding was received for this study.

\section{Conflict of interest}

The authors report no conflict of interest.

\section{References}

1. World Health Organisation. Antibiotic resistance. 2020. Available at https://www.who.int/news-room/factsheets/detail/antibiotic-resistance (accessed October 2020).

2. Wordley V, Shah S, Thompson W. Increased antibiotics use. Br Dent J 2020; 229: 266

3. Hurley S Neligan M. COVID-19 Dental Preparedness Letter. 2020. Available at https://madeinheene.hee.nhs. uk/Portals/0/Dental\%20preparedness $\% 2$ letter $\% 20$ 15\%20April.pdf (accessed October 2020).

4. Hurley S, Neligan M. Resumption of dental services in England. 2020. Available at https://www. england.nhs.uk/coronavirus/wp-content/uploads/ sites/52/2020/03/Urgent-dental-care-letter-28-May. pdf (accessed October 2020).

5. House of Commons Health and Social Care Committee. Delivering core NHS and care services during the pandemic and beyond. 2020. Available online at https://committees.parliament.uk/work/277/ delivering-core-nhs-and-care-services-during-thepandemic-and-beyond/ (accessed October 2020).

6. Cope A L, Francis N A, Wood F et al. Antibiotic prescribing in UK general dental practice: a crosssectional study. Community Dent Oral Epidemiol 2016; 44: 145-153.
7. Suda K, Calip G, Zhou J et al. Assessment of the appropriateness of antibiotic prescriptions for infection prophylaxis before dental procedures 2011 to 2015. JAMA Netw Open 2019; DOI: 10.1001/ jamanetworkopen.2019.3909.

8. SDCEP. Emergency Dental Care: Dental Clinical Guidance. 2007. Available at https://www.sdcep.org. uk/wp-content/uploads/2013/03/EDC+Guidance.pdf (accessed October 2020).

9. Lockhart P B, Tampi M P, Abt E et al. Evidence-based clinical practice guideline on antibiotic use for the urgent management of pulpal-and periapical-related dental pain and intraoral swelling: A report from the American Dental Association. J Am Dent Assoc 2019; DOI: 10.1016/j.adaj.2019.08.020

10. Faculty of General Dental Practitioners (UK). Antimicrobial prescribing for general dental practitioners. 2012. Available online at https://www. fgdp.org.uk/guidance-standards/antimicrobialprescribing-gdps (accessed October 2020).

11. Agnihotry A, Gill K S, Stevenson III R G et al. Irreversible Pulpitis - A Source of Antibiotic Over-Prescription? Braz Dent J 2019; 30: 374-379.

12. Chopra R, Merali R, Paolinelis $G$ et al. An audit of antimicrobial prescribing in an acute dental care department. Prim Dent J 2014; 3: 24-29.

13. Ihimekpen A, Thompson W. Antimicrobial resistance: Antimicrobial prescribing: the work continues. Br Dent J 2018; 225: 3

14. Sarmiento M A, Maglutac Jr M T, Yanga-Mabunga M S Antibiotic Prescribing Practices of Filipino Dentists. Int J Public Health Sci 2019; 8: 332-340.

15. UK Government. Dental antimicrobial stewardship: toolkit. 2016. Available online at https://www.gov.uk/ guidance/dental-antimicrobial-stewardship-toolkit (accessed December 2019).

16. Thornhill M H, Dayer M J, Durkin M J et al. Oral antibiotic prescribing by NHS dentists in England 2010-2017. Br Dent J 2019; 227: 1044-1050.

17. National Health Service Business Services Authority. DR1242. Personal correspondence

18. National Health Service Business Services Authority. DR1242, IR0313 and IR0318. Personal correspondence.

19. The National Archives. Open Government Licence for Public Sector Information. Available at http://www. nationalarchives.gov.uk/doc/open-government-licence/ version/3/ (accessed October 2020).

20. Office for National Statistics. Population Estimates. Available online at https://www. ons.gov.uk/peoplepopulationandcommunity/ populationandmigration/populationestimates (accessed December 2019).

21. Thompson W, McEachan R, Pavitt S et al. Clinician and Patient Factors Influencing Treatment Decisions: Ethnographic Study of Antibiotic Prescribing and
Operative Procedures in OutofHours and General Dental Practices. Antibiotics (Basel) 2020; DOI: 10.3390/ antibiotics 9090575

22. Teoh L,Stewart K, Marino R, McCullough M. Perceptions, attitudes and factors that influence prescribing by general dentists in Australia: a qualitative study. J Oral Pathol Med 2019; 48: 647-654.

23. NICE. Antimicrobial stewardship: Quality Standard (QS121). 2016. Available at https://www.nice.org.uk/ guidance/qs121/resources/antimicrobial-stewardshippdf-75545353537477 (accessed October 2020).

24. Wilson W, Taubert K A, Gewitz M et al. Prevention of infective endocarditis: guidelines from the American Heart Association: a guideline from the American Heart Association Rheumatic Fever, Endocarditis and Kawasaki Disease Committee, Council on Cardiovascular Disease in the Young, and the Council on Clinical Cardiology, Council on Cardiovascular Surgery and Anaesthesia, and the Quality of Care and Outcomes Research Interdisciplinary Working Group. J Am Dent Assoc 2008; 139 Suppl: 3S-24S

25. Oral and Dental Expert Group. Therapeutic Guidelines: Oral and Dental. Version 3. Melbourne: Therapeutic Guidelines Limited, 2019.

26. Thornhill M H, Dayer M J, Prendergast B et al. Incidence and nature of adverse reactions to antibiotics used as endocarditis prophylaxis. J Antimicrob Chemother 2015; 70: 2382-2388

27. NICE. Prophylaxis against infective endocarditis: antimicrobial prophylaxis against infective endocarditis in adults and children undergoing interventional procedures. 2016. Available at https://www.nice.org.uk/guidance/cg64/resources/ prophylaxis-against-infective-endocarditisantimicrobial-prophylaxis-against-infectiveendocarditis-in-adults-and-children-undergoinginterventional-procedures-pdf-975567956677 (accessed October 2020)

28. Teoh L, Marino R J, Stewart K, McCullough M. A survey of prescribing choices of general dentists in Australia. BMC Oral Health 2019; 19: 193

29. Buttar R, Aleksejuniene J, Coil J. Antibiotic and Opioid Analgesic Prescribing Patterns of Dentists in Vancouver and Endodontic Specialists in British Columbia. J Can Dent Assoc 2017; 83: h8.

30. Sturrock A, Landes D, Robson T et al. An audit of antimicrobial prescribing by dental practitioners in the north east of England and Cumbria. BMC Oral Health 2018; 18: 206.

31. British Dental Association. Dentists press for funded urgent time to deliver Hancock's vision on antibiotics. 2019. Available at https://www.bda.org/news-centre/ press-releases/Pages/Dentists-press-for-fundedurgent-time-to-deliver-Hancock-vision-on-antibiotics. aspx (accessed October 2020). 